\title{
Fabrication and Nanostructured Surface Layers of Al Alloy by Surface Vibrational Mechanical Attrition
}

\author{
X. Wu ${ }^{1}$, Y. Hong ${ }^{1}$, J. Lu ${ }^{2}$, and K. Lu ${ }^{3}$ \\ ${ }^{1}$ State Key Lab of Nonlinear Mechanics, Inst of Mech, Chinese Acad of Scis, Beijing, China \\ ${ }^{2}$ LASMIS, Univ of Tech of Troyes, 10000, Troyes, France \\ ${ }^{3}$ Shenyang National Lab for Mater Sci, Inst of Metal Rsrch, Chinese Acad of Scis, Shenyang, \\ China
}

\begin{abstract}
Nanograins were introduced into the surface layers of an Al-alloy during surface vibrational mechanical attrition. Transmission electron microscopy revealed that microstructures developed with an increase in strain, in the following sequence, i.e., lamellar microbands of elongated subgrains, equiaxed submicro-, and nano-grains respectively. The grain subdivision into the subgrains was found to be the main mechanism responsible for grain refinement. The simultaneous evolution of high boundary misorientations was ascribed to the boundary rotation for accommodating further strains. Results showed that the grains could refine remarkably into the nanometer regime $(<100 \mathrm{~nm})$ within the outer surface of the layer. The depth of the nanocrystal layers increased and the grain size decreased with the extension of processing time. The nanocrystal layers evidenced an increase in hardness.
\end{abstract}

\section{INTRODUCTION}

Synthesis of the nanostructured layers is of current interest, on account of unusual improvements of the surface-related properties including hardness, fatigue, wear and friction, corrosion etc [1-4]. By means of surface mechanical processing techniques, nanostructured surface layers can be created in metallic materials [2]. In particular, the mechanical treatment offers two significant advantages over other coating and deposition methods. First, the nanograins are free from residual porosity and impurities. Second, the chemical composition and the physical dimension remain intact on the surface. Recent studies $[5,6]$ have shown that the ultrasonic shot peening processing can produce nanostructured surface layers in 316L stainless steel and pure iron. In the present study, we have investigated the microstructures in the surface layer of an $\mathrm{Al}$ alloy produced by surface vibrational mechanical attrition (SVMA), with the aim to understand the relation of microstructural evolution to the successive increase in strain and to elucidate the underlying mechanism for the nanograin formation.

\section{EXPERIMENTAL DETAILS}

The experimental material was an $\mathrm{Al}$ alloy 7075 , with a composition (wt pct) of $2.5 \mathrm{Mg}, 5.6 \mathrm{Zn}$, $2.2 \mathrm{Cu}, 0.23 \mathrm{Cr}$, balance Al. The initial grain size was determined to be $\sim 80 \mu \mathrm{m}$. A commercially available plate was cut into pieces with $100 \times 100 \times 10 \mathrm{~mm}^{3}$ in dimensions.

The principle of the SVMA technique is as follows. A high-energy generator vibrated the reflecting chamber, where the stainless steel shots of $7.5 \mathrm{~mm}$ diameter resonated. The shots then caused repetitive, high-speed, and multi-directional impact onto the surface of materials. Resultantly, severe strains with a strain gradient changing from zero far in the matrix to the 
maximum at the top surface were imparted into the surface by contact loading. Details of the equipment were reported elsewhere [2,5]. SVMA processing was conducted under the vacuum at the room temperature for 5,10 , and 15 minutes respectively.

Transmission Electron microscopy (TEM) was carried out using a JOEL-2010 operating at 200 $\mathrm{kV}$. Thin TEM films were prepared as follows: i) by sticking a castolite plate of $2 \mathrm{~mm}$ thick on the affected surface, ii) by cutting a bar of $3 \mathrm{~mm}$ in diameter with the layer located in the middle, iii) by cutting thin discs of $30 \mu \mathrm{m}$ thick using a diamond saw normal to the long axis of the bar, and iv) by dimpling and argon-ion-beam thinning to perforation at the room temperature. This method allowed the examination of a well-identified depth of the processed surface layer.

Measurements of grain sizes are made directly from TEM micrographs and the reported values are the averages from 40-60 individual measurements. Because of the elongated nature of the subgrains, the datum points were separately presented for measurements of the average of the short axis and the average of the long axis, whereas measurements for the grains were taken consistently along randomly selected directions.

Hardness measurements were performed at the cross-section of the processed layer using a Vickers hardness tester under a $200 \mathrm{~g}$ load applied for 50 seconds. The reported values were the averages of 5 measurements.

\section{Results}

\section{$\underline{\text { Microstructure }}$}

To investigate the microstructural evolution and grain refinement, deformation structures at a large strain (processed for $15 \mathrm{~min}$ ) were studied and are shown in Figure 1.

Two prominent deformation features are seen at the initial stage (low strain), in the area next to the strain-free matrix ( $62 \mu \mathrm{m}$ deep from the top surface). First, as shown in Figure 1(a), particles of the dispersely distributed second phase could act as an emission source releasing a great number of dislocations. The particle is determined to be $\mathrm{Al}_{2} \mathrm{Cu}$ according to its electron diffraction pattern (EDP). Secondly, many dislocation cells (Figure 1 (b)) present are an evidence for severe dynamic straining.

Figure 1(c) shows that the lamellar microbands (MBs) of elongated subgrains develop with an increasing strain $(\sim 59 \mu \mathrm{m}$ deep from the top surface). Extended MBs are interestingly found to be $\sim 0.4-1.0 \mu \mathrm{m}$ wide but as long as 3-15 $\mu \mathrm{m}$. MBs have various orientations, due to the change of the strain path. In the inset is the EDP showing an undeveloped circle with well-defined diffraction spots, indicating that MBs consist of low angle misorientations.

Figure 1(d) reveals the grain subdivision to subgrains and the evolution of boundary misorientations inside the interior of a larger grain ( $\sim 0 \mu \mathrm{m}$ deep from the top surface). Eleven small subgrains of various sizes (denoted by large size digits inside the subgrain interior) are present. Boundary misorientations between neighboring subgrains were also determined (denoted by small size digits at grain boundaries). It is interesting to note that encircling subgrain 1 , three remarkably different boundary morphologies are noted. The left side, also a portion of original larger grain, is sharp with high boundary misorientation of $12.8^{\circ}$. Extinction contours with boundary misorientation of $7.8^{\circ}$ appear in the boundary adjacent to subgrain 11 , indicating the presence of high internal stresses and non-equilibrium due to the presence of a high density of dislocations at the boundaries [7]. The boundary abutting subgrain 2 consists of an undeveloped dislocation wall, with boundary misorientation of just $0.8^{\circ}$. 

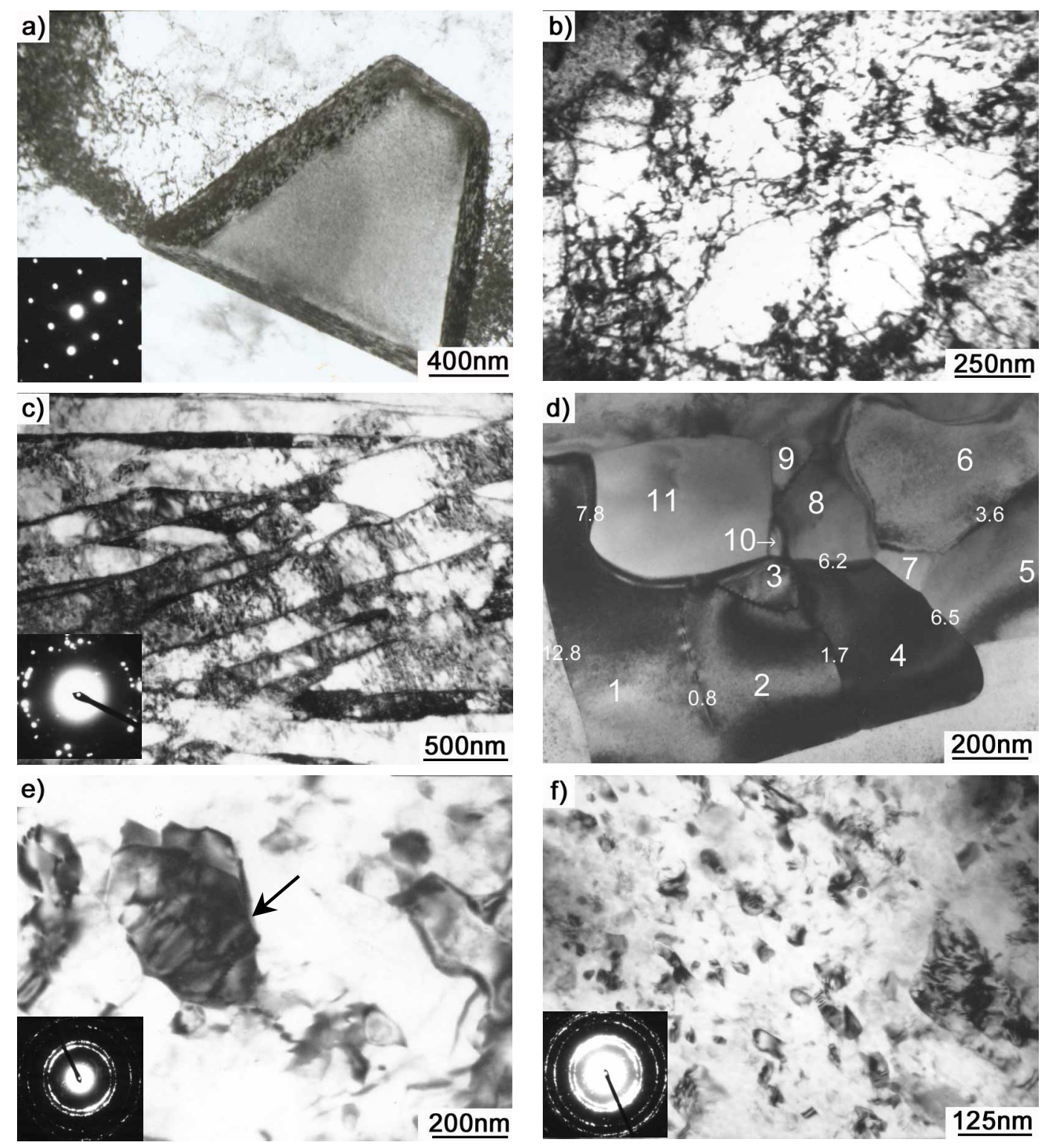

Figure 1. Microstructures at various layer depths after 15 min processing (a) dislocation emission source, (b) dislocation tangles and cells, (c) extended microbands of elongated subgrains, (d) grain subdivision, (e) submicrograins, and (f) nanograins.

Figure 1(e) is a TEM micrograph showing equiaxed, submicro-sized grains as the strain increases ( $35 \mu \mathrm{m}$ deep from the top). The further subdivision of grain into smaller subgrains of $\sim$ $100 \mathrm{~nm}$ in diameter are seen inside one large grain of $\sim 300 \mathrm{~nm}$ in diameter (denoted by arrow). Most grain boundaries are not well defined, indicating the presence of high internal stresses, consistent with those formed using other severe plastic deformation processes [8]. Inset is the ring-like EDP, indicating that there are many small grains with highly misoriented boundaries in the selected area of view. 
Figure 1(f) reveals equiaxed, nanograins ( $\sim 5 \mu \mathrm{m}$ deep from the top surface). Inset is the ring-like EDP, indicating high boundary misorientations.

\section{Depth and grain size of nanocrystal layers}

Figure 2(a) shows the variation of layer depth with processing times. It is seen that the whole affected layer consists of three parts, containing nanograins $(<100 \mathrm{~nm})$ in the outer surface, submicrograins (0.1-1 $\mu \mathrm{m})$ in the sub-surface of the layer and MBs next to the strain-free matrix respectively, from the top surface to inside. The longer the processing time, the deeper is both the nanocrystal layer depth and the whole affected layer depth.

Figure 2(b) shows the variation of grain size as a function of depth with various processing times. Note that grains refine evidently from inside to the surface. And, the longer the processing time, the finer are the grains.

\section{Microhardness of surface layer}

Figure 3 shows the variation of microhardness with depth for different processing times. It is seen that all the curves showed a dramatic increase in hardness from inside to layer surface. The hardness increases with extension of the processing time.

\section{DISCUSSION}

According to the microstructural observation (Figure 1), nanograins are created in the surface layer during SVMA. The development of equiaxed, stable grains consists of two steps, i.e., the subdivision of grains and the evolution of highly misoriented boundaries. Intense strains are imparted into the surface layer during SVMA. The mechanism responsible for accommodating large amounts of strain is to subdivide original grains into subgrains [9-12]. The subdivision of grains takes place with increasing strain and the extended MBs of subgrains, submicro- and nano-grains are produced.
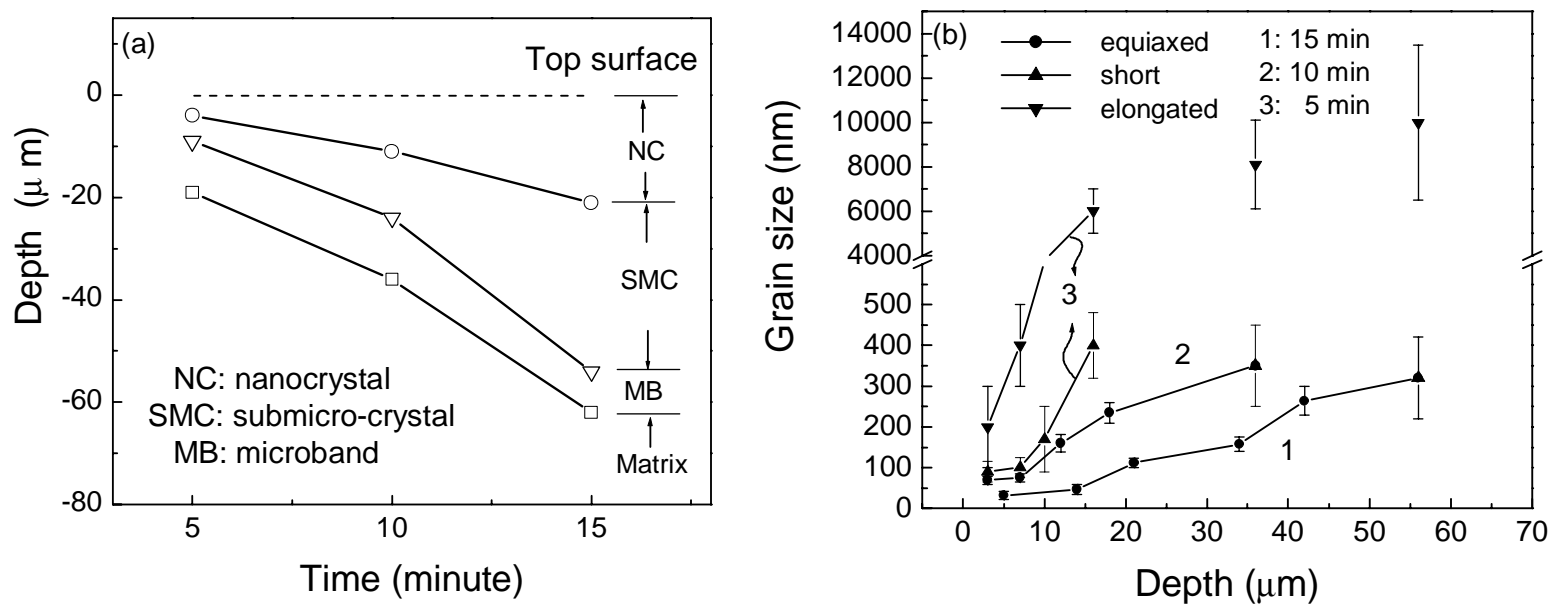

Figure 2. Variation of (a) layer depth with processing time and (b) grain size with depth. 


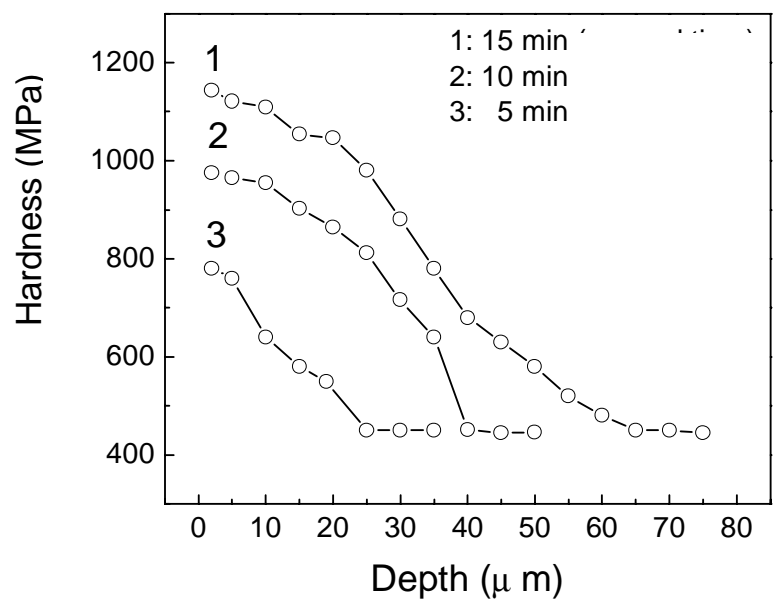

Figure 3. Variation of hardness with depth and various processing times

According to Figure 1(d), the evolution of boundary misorientations is historically dependent on strain. Subgrains form with certain dislocation patterns becoming their boundaries such as dislocation walls (boundary between subgrains 1 and 2) at the initial stage of low strain. An increase in strain results in an increase in dislocation density and decrease in spacing in walls (boundary between subgrains 2 and 3 ). After the deformation is completed, no additional dislocations are being added to the boundaries and then the subgrain boundary refinement creates the facet, high angle boundaries (boundaries between grains 4 and 5) by reducing the excess dislocation dipoles through dislocation annihilation by dislocation climb. At this stage, since the dislocation movement is more strongly restricted, slip systems within adjacent subgrains will be activated in response to applied straining in order to make subgrain orientation more energetically favorable [9]. According to the measured boundary misorientations and boundary morphologies, it is reasonably deduced that the sub-boundary, low- and high-angle boundaries developed successively with increasing strain. In other words, the grain refinement is simultaneously accompanied by an increase in misorientation across subgrain boundaries. SVMA provides the multi-directional strain path and high strain rate, which are especially effective at promoting subgrain rotation. Therefore, the accumulated rotation of subgrains appears to be the primary mechanism as a means of accommodating further deformation, resulting in highly misoriented, equiaxed grains.

Note that a high density of dislocations often accompanies the subgrain formation. Dislocation patterns begin to rotate and high angle boundaries slide and cause rotation of the closest subgrains that acquire high angle boundaries and begin in turn to slide. With increasing strain, more dislocations are generated and contribute to rotate. The additional slip systems may be triggered and the portion of high-angle misorientations increase. The large numbers of dislocations that are present in the subgrain boundaries can accommodate the rotation sufficiently to prevent gaps and cavities from forming when subgrains rotate away from each other (i.e., there are sufficient, geometrically necessary dislocations present to accommodate these rotations.)

SVMA produces multi-directional straining which leads to the change of slip systems with the strain path even in the interior of the same subgrain. The dislocations not only interact with other dislocations in the current active slip systems, but also interact with inactive dislocations generated in previous straining. This will promote the grain subdivision. As a consequence, the effectiveness of grain refinement is enhanced. 
The depth of nanocrystal layer increases with the processing time (Figure 2(a)). This is due to the reduced route for both dislocation diffusion and strain transfer during the grain refinement. The longer the processing time, the larger is strain imposed into the layer. The grain subdivision goes to a larger extent. Therefore, the grains refine most remarkably with processing time of $15 \mathrm{~min}$ (Figure 2(b)). The increase in hardness in surface layer is mainly due to the grain refinement (Figure 3). And, the finer the grains, the higher is the hardness.

\section{CONCLUSIONS}

This study shows how the nanocrystal grains develop with an increased strain into the surface layer of an Al alloy during surface vibrational mechanical attrition. The microstructural evolution is characterized by the successive grain subdivision and simultaneous increment of boundary misorientations. There is an increase in the depth of affected layer, a decrease in grain size with a consequent increase in hardness with the extension of processing time.

\section{ACKNOWLEDGMENTS}

This research was supported by National Natural Science Foundation of China and The Chinese Academy of Sciences. One of authors (X.W.) thanks Dr. C. Ramachandra for his critical reading of this manuscript.

\section{REFERENCES}

1. H. Gleiter. Acta Mater. 48, 1 (2000).

2. K. Lu and J .Lu, J. Mater. Sci. Technol. 15, 193 (1999).

3. B. Canton. Mater. Sci. Forum 307, 143 (1999).

4. V. L. Tellkamp, M. L. Lau, A. Fabel and E. J. Lavernia. Nanostr. Mater. 9, 489 (1995).

5. N. R. Tao, M. L. Sui, J .Lu, and K. Lu, Nanostru. Mater. 11, 433 (1999).

6. G. Liu, J. Lu and K. Lu. Mater. Sci. Engng. A286, 91 (2000).

7. R. Z. Valiev, R. K. Islamgaliev, and I. V. Alexandrov, Prog. Mater. Sci. 45, 103 (2000).

8. Y. Iwahashi, Z. Horita, M. Nemoto, and T. G. Langdon, Acta Mater. 45, 4733 (1997).

9. B. Bay, N. Hansen, D. A. Hughes, and D. Kuhlmann-Wilsdorf, Acta Mater. 40, 205 (1992).

10. D.A. Hughes, D.C. Chrzan, Q. Liu and N. Hansen. Phy.Rev. Lett. 81, 4664 (1998).

11. N. Hansen, X. Huang and D. A. Hughes. Mater Sci Engng. A317, 3 (2001).

12. N. Hansen and D. A. Hughes. Phys. Status Solidi (b). 149, 155 (1995). 